Check for updates

Cite this: RSC Adv., 2019, 9, 9193

Received 18th December 2018

Accepted 2nd March 2019

DOI: $10.1039 / c 8 r a 10373 a$

rsc.li/rsc-advances

\title{
Green synthesis and in situ immobilization of gold nanoparticles and their application for the reduction of $p$-nitrophenol in continuous-flow mode
}

\author{
Rózsa Szücs, ${ }^{\text {ab }}$ Diána Balogh-Weiser, ${ }^{c d}$ Evelin Sánta-Bell, ${ }^{c}$ Eszter Tóth-Szeles, ${ }^{b}$ \\ Tamás Varga, ${ }^{e}$ Zoltán Kónya, (D) ${ }^{\text {ef }}$ László Poppe (D) ${ }^{\mathrm{cg}}$ and István Lagzi (D)*bh
}

A green and facile method has been developed for the preparation of in situ immobilized gold nanoparticles (AuNPs) using agarose as a reducing and stabilizing agent. The size of the synthesized AuNPs ranges between 10 and $100 \mathrm{~nm}$, and their average size can be controlled by the concentrations of the agarose and gold salt. The agarose matrix as a mild and green reaction medium can provide a good dispersion environment for forming AuNPs, and the hydrogel can be well homogenized with polyacrylic macroporous microbeads as well, which can adsorb and stabilize the particles leading to the simultaneous synthesis and immobilization of AuNPs avoiding harmful inorganic compounds or organic solvents. The supported gold nanocatalyst was successfully applied as a catalyst in packed bed reactors for efficient $\mathrm{NaBH}_{4}$-mediated reduction of $p$-nitrophenol in continuous-flow mode.

\section{Introduction}

Nowadays, gold nanoparticles (AuNPs) are being extensively used as efficient redox catalyst materials. ${ }^{1-7}$ There has been an explosion in the number of reactions for which AuNPs have shown exceptional efficacy. ${ }^{8}$ Nanoparticles (NPs) are usually thermodynamically unstable, and hence do not possess good long-term stability, primarily as a result of the aggregation due to van der Waals attraction. ${ }^{9}$ Therefore, establishing and maintaining stability is of particular importance in order to use NPs in catalysis. The long-term stabilization can be reached by

${ }^{a}$ MTA-BME Computer Driven Chemistry, Budapest University of Technology and Economics, H-1111, Szent Gellért tér 4, Budapest, Hungary

${ }^{b}$ Department of Physics, Budapest University of Technology and Economics, 1111, Budafoki út 8, Budapest, Hungary

'Department of Organic Chemistry and Technology, Budapest University of Technology and Economics, H-1111, Szent Gellért tér 4, Budapest, Hungary

${ }^{d}$ Department of Physical Chemistry and Materials Science, Budapest University of Technology and Economics, H-1111, Budafoki út 8, Budapest, Hungary

${ }^{e}$ Department of Applied and Environmental Chemistry, University of Szeged, $H-6720$, Rerrich Béla tér 1, Szeged, Hungary

${ }^{f}$ MTA-SZTE Reaction Kinetics and Surface Chemistry Research Group, H-6720, Rerrich Béla tér 1, Szeged, Hungary

${ }^{g}$ Biocatalysis and Biotransformation Research Centre, Faculty of Chemistry and Chemical Engineering, Babeș-Bolyai University of Cluj-Napoca, Arany János Str. 11, RO-400028 Cluj-Napoca, Romania

${ }^{h}$ MTA-BME Condensed Matter Research Group, Budapest University of Technology and Economics, H-1111, Budafoki út 8, Budapest, Hungary. E-mail: istvanlagzi@gmail. com; Fax: +361463 3567; Tel: +3614631341 two main methods, either based on steric hindrance, therefore using bulky protecting groups, or based on electrostatic repulsion between ligands bearing the same charge. ${ }^{\mathbf{1 0 1 1}}$ Both stabilization methods have their own advantages and drawbacks, the steric stabilization is often effective and sustainable, however, the stabilizing agents can have a negative effect on the morphology of the formed particles or on the accessibility of the surface, leading to a decrease in catalytic activity. Stabilizing throughout electrostatic interaction is rather straightforward, as it can be fine-tuned by adjusting the concentration of the ionic species. ${ }^{12}$ The possible use of AuNPs as catalysts has attracted vast research interest, focusing on their unique properties that can be linked to their particle size and shape.

As an alternative to classical batch methods, organic reactions carried out in continuous-flow reactors have recently attracted much attention in synthetic organic chemistry. ${ }^{\mathbf{1 3}}$ These reaction systems can offer safer, more practical, furthermore more sustainable means for the preparation of valuable organic compounds. In such systems, the use of immobilized catalysts can further improve the green aspect of the reactions.

In this work, we present a novel green and one-step method for the synthesis and immobilization of AuNPs in the agarose matrix (AuNPs-AR). The combination of agarose gel and solid macroporous polymer beads (AuNPs-AR-P) provides a stable and easy-to-handle catalyst for the reduction of organic compounds in continuous-flow mode using packed bed reactor. To prove the concept of the applicability, we 

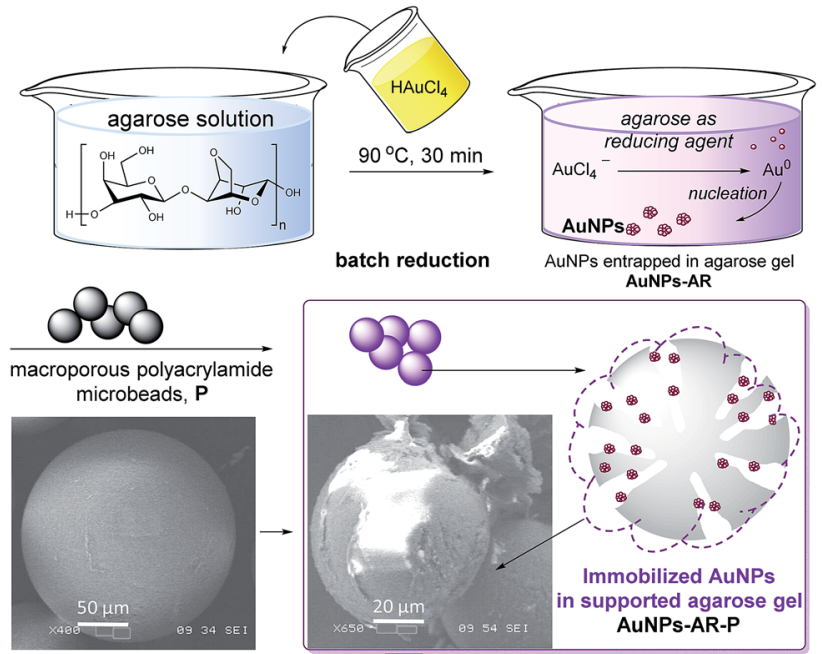

batch reduction

AuNPs entrapped in agarose gel AuNPs-AR

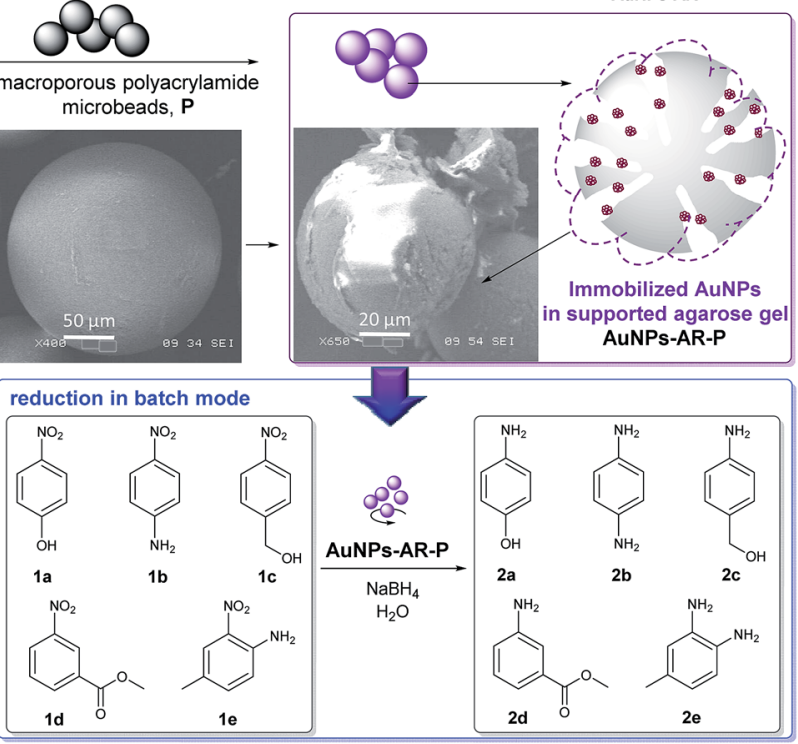

packed bed reactor in continuous-flow mode
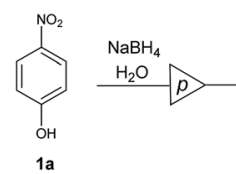

1a

(1)

Fig. 1 In situ synthesis and immobilization of AuNPs in agarose combined with macroporous polymer beads (AuNPs-AR-P) and the application of Au-AR-P as an immobilized catalyst in reduction of $1 \mathrm{a}-\mathrm{e}$ in batch mode and in a packed bed reactor for continuous-flow reduction of $p$-nitrophenol (1a).

performed the reduction of $p$-nitrophenol to $p$-aminophenol by sodium borohydride $\left(\mathrm{NaBH}_{4}\right)$ catalyzed by AuNPs-AR-P system (Fig. 1).

\section{Experimental section}

\subsection{Synthesis and immobilization of gold nanoparticles using agarose}

AuNPs were synthesized using agarose (Type I Agarose, SigmaAldrich) and gold(III) chloride trihydrate (Sigma-Aldrich) at various concentrations of both reagents in water. A solution of agarose $(4.5 \mathrm{~mL}$ water, $0.025 \mathrm{~g}$ to $0.150 \mathrm{~g}$ agarose, leading to 0.5 and $3.0 \mathrm{~m} / \mathrm{v} \%$ concentrations calculated for the $5 \mathrm{~mL}$ overall solution) was heated to $90^{\circ} \mathrm{C}$ and vigorously stirred in a heating magnetic stirrer at $400 \mathrm{rpm}$. When the solution of agarose became transparent (after $15 \mathrm{~min}$ of heating), a solution of the gold salt $(0.5 \mathrm{~mL}$, with concentration ranging between 0.10 and $10.0 \mathrm{mM}$, leading to final concentrations between 0.01 and 1.0 $\mathrm{mM}$ ) was added to the stirred hot solution. After keeping the mixture at $90{ }^{\circ} \mathrm{C}$ for 30 min with a continuous stirring, it was allowed to cool down to room temperature (RT) to result in a colored hydrogel.

\subsection{Characterization of synthesized gold nanoparticles}

UV-Vis spectra were recorded at RT using a UV-1600PC spectrophotometer (VWR). All transmission electron micrographs were acquired via a FEI TECNAI G2 20 X-Twin high-resolution transmission electron microscope (HRTEM) operated at 200 $\mathrm{kV}$ accelerating voltage.

\subsection{The preparation of the catalyst mixture in batch mode}

An agarose-gold solution mixture (10 mL: $1.0 \mathrm{~m} / \mathrm{v} \%$ agarose and $1.0 \mathrm{mM}$ gold salt) was prepared as described above. After cooling to RT, $400 \mathrm{mg}$ of ethylamine-functionalized macroporous polymer resins (RelizymeTM EA 403; polymethyl methacrylate beads, particle size $150-300 \mu \mathrm{m}$, pore size $400-600 \AA$ A purchased from Resindion S.r.l. Rome, Italy) were added to AuNPs-AR as immobilization carrier. The mixture was homogenized by mechanical stirring, then it was centrifuged for 10 minutes at 330 RCF (RT) to recover the formed AuNPs-AR-P beads. After washing with ethanol, the beads were dried at room temperature. For reduction of nitro compounds 1a-e in batch mode, AuNPs-AR-P $(5 \mathrm{mg})$ was added to the mixture of nitro compound (1a-e; $3 \mathrm{mM} ; 10 \mathrm{~mL}$, in $\mathrm{H}_{2} \mathrm{O}$ in case of $\mathbf{1 a}, \mathbf{b}$, $\mathbf{c}$, e and in $\mathrm{H}_{2} \mathrm{O}$ / ethanol 9/1 v/v in case of $1 \mathrm{~d})$ with $\mathrm{NaBH}_{4}(2.0 \mathrm{M}$ in triethylene glycol dimethyl ether, 25 fold excess) and the reaction mixture was shaken at room temperature. Samples were analyzed by a UV-VIS spectrophotometer measuring the spectra from $\lambda=$ 200 to $600 \mathrm{~nm}$.

\subsection{Preparation of cartridge for continuous-flow experiments}

The AuNPs-AR-P beads described in 2.3 were packed into stainless steel CatCart ${ }^{\mathrm{TM}}$ columns (inner diameter: $4 \mathrm{~mm}$; total length: $70 \mathrm{~mm}$; packed length: $65 \mathrm{~mm}$; inner volume: $0.816 \mathrm{~mL}$ ) according to the filling process of ThalesNano Inc. The columns were settled by silver metal (Sterlitech Silver Membrane from Sigma-Aldrich, Z623237, pore size: $0.45 \mu \mathrm{m}$; pure metallic silver, 99.97\% with no extractable or detectable contaminants) and PTFE (Whatman® Sigma-Aldrich, WHA10411311, pore size: $0.45 \mu \mathrm{m})$ filter membranes. The sealing units were made of PTFE (filling weight: $150 \mathrm{mg}$ ) and used in a flow reactor operated in continuous-flow mode.

\section{Results and discussion}

In several wet synthesis methods for the production of AuNPs, the reducing and the capping/stabilizing agents are the same (e.g., citrate, ${ }^{14}$ amino acids ${ }^{15}$ and gelatin ${ }^{16}$ ). In our approach, we used agarose as a reducing agent, and the agarose and the formed hydrogel matrix stabilized the formed AuNPs. In most studies published in the literature, the agarose gel has been primarily used as a matrix for the pre-prepared AuNPs, which were synthesized by others reducing agents (trimeric alanine phosphine conjugate, sodium borohydride and sodium 
citrate). ${ }^{17,18}$ Only one study utilized agarose monomers as a reducing agent and used those AuNPs further as a template to produce a porous nano-hybrid material. ${ }^{19}$ No detailed investigation has been provided on the effect of the agarose on the formed AuNPs, and no example has been shown to use this hybrid hydrogel for catalytic purposes.

The effect of the concentrations of gold salt $\left(\mathrm{AuHCl}_{4} \times 3 \mathrm{H}_{2} \mathrm{O}\right)$ and agarose was systematically studied on the synthesis of AuNPs. After the synthesis process, the samples had various distinct colors: red, purple, or blue depending on the concentrations of the reagents (Fig. 2). This indicates that the generated AuNPs have various average sizes with various polydispersity. Fig. 3 presents this fact in a more qualitative way showing the observed wavelength of maximum absorption peak in regard to the concentration of the reactants. At fixed agarose concentrations, the wavelength of the maximum absorption peak $\left(\lambda_{\max }\right)$ shifts toward higher wavelengths with the increasing concentration of the gold precursor, indicating the formation of larger AuNPs. Due to the surface plasmon resonance (SPR) of the metal core of the particles, the wavelength of the maximum absorption peak in the UV-Vis spectra of AuNPs correlates with the size of the particles. Samples containing small $(\sim 5-10 \mathrm{~nm})$ and monodisperse $(<15 \%)$ AuNPs have $\lambda_{\max }$ around $520 \mathrm{~nm}$ (having red color), both bigger size and more polydispersity of the AuNPs



Fig. 2 Effect of the agarose and Au precursor concentration on the colour of the formed and in situ immobilised AuNPs in agarose matrix (AuNPs-AR)

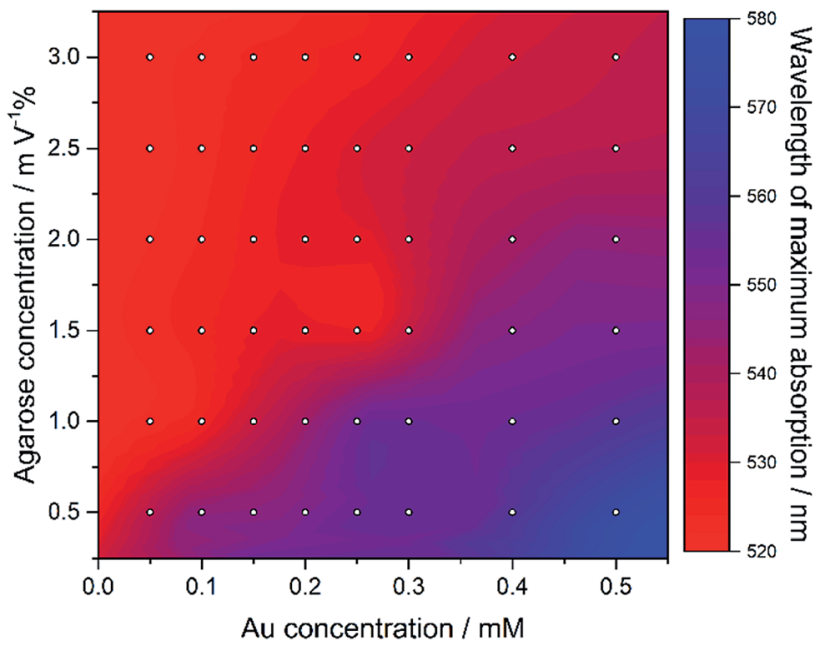

Fig. 3 Effect of the concentrations of the agarose and the gold salt on the wavelength of maximum absorption $\left(\lambda_{\max }\right)$ of the UV-Vis spectra of the formed agarose gels containing AuNPs (AuNPs-AR).

generate greater $\lambda_{\max }$ values (Fig. 3). Higher agarose concentration provides particles with a smaller size (lower wavelength of the maximum absorption peak). Transmission electron microscopy (TEM) fully supports these findings (Fig. 4), namely the average size of the AuNPs (and the polydispersity of the particles) can be fine-tuned by the initial concentrations of the agarose (reducing agent) and the gold salt. ${ }^{20}$ Our result is in good accordance with a finding that higher concentration of a gold salt provides larger particles with higher polydispersity. ${ }^{21-23}$ Interestingly, AuNPs-AR are stable for several weeks after the synthesis. Agarose gel is macroporous (pore diameter $\sim 100-200 \mathrm{~nm}$ ) with large inner surface area, ${ }^{24}$ which provides sites for nucleation and growth. ${ }^{25,26}$

These sites immobilize particles in the gel matrix, therefore preventing them from further aggregation. In other words, this

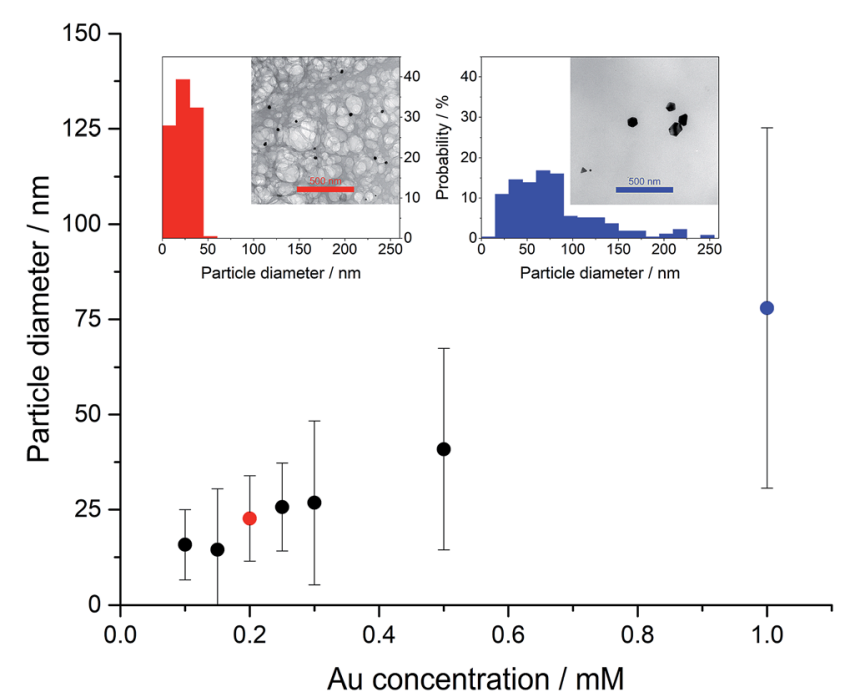

Fig. 4 The average size of the AuNPs, formed at agarose concentration of $2.0 \mathrm{~m} / \mathrm{v} \%$ and various concentrations of gold salt, as determined by transmission electron microscopy (TEM). Insets show TEM micrographs and size distributions of two selected samples indicated by red and blue colours. 
technique does not require the use of external capping (stabilization) agents. Even though agarose gel matrix is an effective agent for the fine-tunable synthesis and immobilization of AuNPs, application of this system in batch mode and in packed bed reactors in continuous-flow mode is unmanageable due to its poor mechanical properties and significant swelling. Thus, macroporous polyacrylamide beads were applied as support for the AuNPs entrapped in agarose gel.

The Au-catalyzed reduction of aromatic nitro compounds 1a-e has high potential, while they are widely used building blocks for the synthesis of bioactive agents. The catalytic activity of noble metal catalysts, can be determined easily by using UVVis spectroscopy. ${ }^{7,27,28}$ To demonstrate the reductive efficiency of our immobilized Au catalyst (AuNPs-AR-P) the nitro group of substrate 1a-e was reduced in the presence of $\mathrm{NaBH}_{4}$ (Fig. 1). First, we examined the blank reaction, in the absence of AuNPsAR-P, in each cases. Since no significant reduced product $\mathbf{2 a - e}$ was observed without Au-catalysis in any case upon mixing the 1a-e and the reducing agent for $24 \mathrm{~h}$, it is safe to conclude that the conversions observed in the presence of AuNPs-AR-P catalyst are due to Au-catalysis (Table 1).

Based on our results in the reduction in batch mode, the resulted stable and easy-to-handle catalyst (AuNPs-AR-P synthesized using $1.0 \mathrm{~m} / \mathrm{v} \%$ agarose and $1.0 \mathrm{mM}$ gold salt) can be used as a filling material in packed bed columns. In the continuous-flow experiments, the AuNPs-AR-P-mediated reduction of $p$-nitrophenol (1a) to $p$-aminophenol (2a) by sodium borohydride $\left(\mathrm{NaBH}_{4}\right)$ used in 10-fold excess under alkaline conditions was investigated as a model reaction. In this set of experiments, the concentrations of substrate 1a and the flow rate were systematically changed and conversions were analyzed (Fig. 5). Conversion was determined as an average of five consecutive measurements after $5 \mathrm{~min}$ intervals at the steady state. The standard deviation of conversions being under $5.0 \%$ (1.6\% in average) indicated the robustness of this system. The effluents investigated in the wavelength region characteristic for AuNPs $(\lambda=523 \mathrm{~nm})$ indicated no leaching of the catalyst in any of the experiments. By varying the concentration and flow rate in the range indicated in Fig. 5, conversions between $17 \%$ and $100 \%$ could be achieved.

As expected, the flow rate as well as the reactant concentration had significant impact on the conversion of the $p$-nitrophenol reduction in continuous-flow experiments. Although the

Table 1 The catalytic efficiency ( $c$ : conversion, $r_{\text {batch: }}$ productivity of the catalyst) of AuNPs-AR-P in the reduction of $1 a-e$ in batch mode

\begin{tabular}{lllll}
\hline & \multicolumn{2}{l}{ Without AuNPs-AR-P } & & \multicolumn{2}{l}{$\begin{array}{l}\text { With AuNPs-AR-P } \\
\text { catalyst }\end{array}$} \\
\cline { 2 - 2 } Substrate 1 & $c^{a} / \%$ & & $c^{b} / \%$ & $r_{\text {batch }} / \mathrm{U}^{b} \times \mathrm{g}^{-1}$ \\
\hline a & $<0.1$ & 56.4 & 332 \\
b & $<0.1$ & 53.0 & 312 \\
c & $<0.1$ & 10.7 & 63 \\
d & $<0.1$ & 11.3 & 67 \\
e & $<0.1$ & 56.5 & 332
\end{tabular}

${ }^{a} c$ was calculated after reaction time 24 h. ${ }^{b} c$ was calculated after reaction time $10 \mathrm{~min}$.
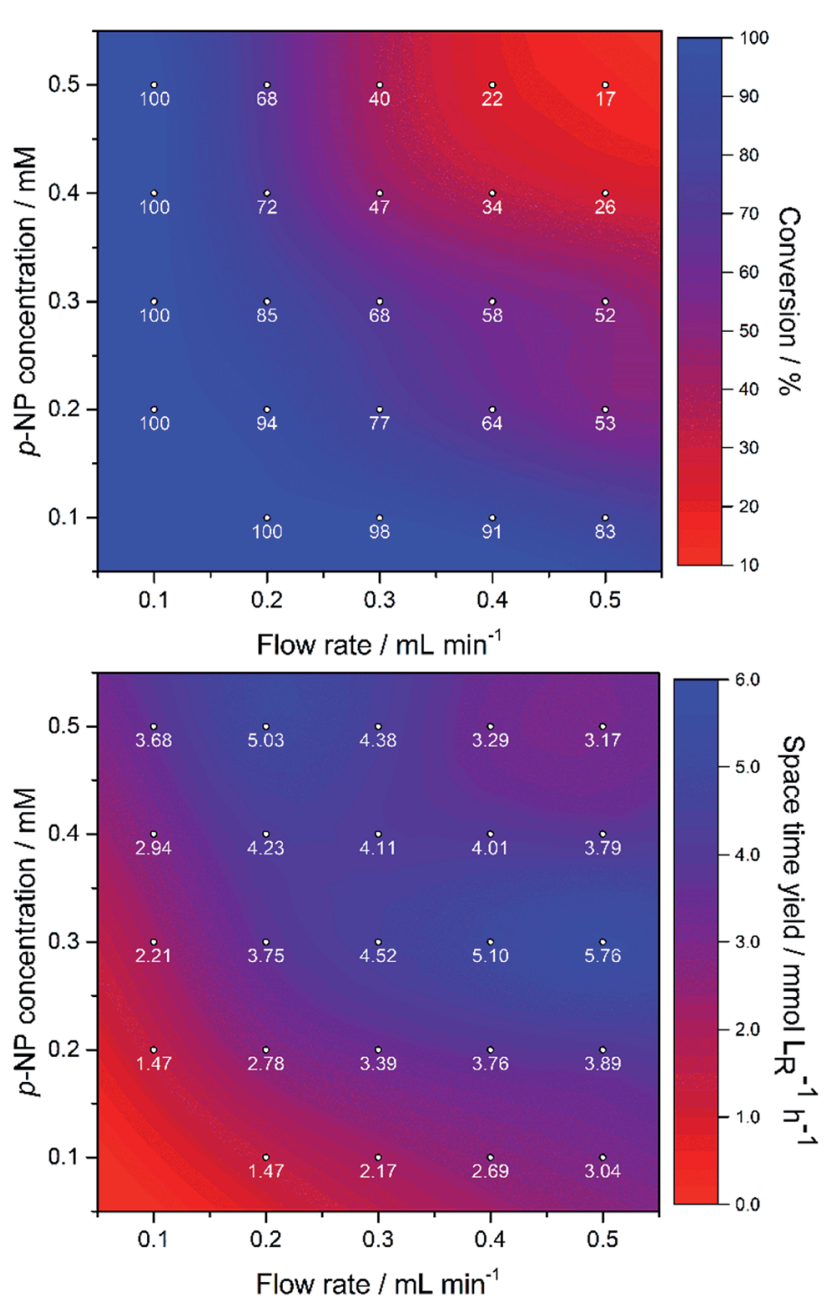

Fig. 5 Catalytic efficiency of AuNPs-AR-P $(1.0 \mathrm{~m} / \mathrm{v} \%$ agarose and $1.1 \mathrm{mM}$ gold salt) characterized by conversion and space time yield of the reduction of $p$-nitrophenol $(p-N P)$ in continuous-flow mode at different substrate concentrations and flow rates.

use of the $p$-nitrophenol $/ p$-aminophenol system is popular to demonstrate the catalytic efficiency of newly formed AuNPs, recirculation of the AuNP catalysts from batch processes which were investigated in most cases, ${ }^{29,30}$ - remains a challenge. By using our flow system, the catalyst is readily reusable after the reaction just by washing the packed bed column with water to remove the lingering reactants

\section{Conclusions}

In summary, we presented a facile and green one-step method for the production of AuNPs using agarose as a reducing and stabilizing agent. The size of the AuNPs and the polydispersity of the samples could be controlled by the concentrations of the initial reagents. We used macroporous polymer beads to support the AuNPs immobilized in the agarose gel enabling Aucatalysis in a packed bed reactor. The catalytic efficiency of this hybrid system was demonstrated in reduction of $p$-nitrophenol by sodium borohydride carried out in continuous-flow mode. 


\section{Conflicts of interest}

There are no conflicts to declare.

\section{Acknowledgements}

Authors acknowledge the financial support of the National Research, Development and Innovation Office of Hungary (NN125752 and K120115) and the BME-Nanotechnology FIKP grant of EMMI (BME FIKP-NAT).

\section{Notes and references}

1 D. T. Thompson, Nano Today, 2007, 2, 40-43.

2 G. J. Hutchings and J. K. Edwards, in Front. Nanosci., ed. R. L. Johnston and J. P. Wilcoxon, Elsevier, 2012, pp. 249-293.

3 A. Corma and H. Garcia, Chem. Soc. Rev., 2008, 37, 20962126.

4 M. Stratakis and H. Garcia, Chem. Rev., 2012, 112, 4469-4506.

5 R. Grisel, K.-J. Weststrate, A. Gluhoi and B. E. Nieuwenhuys, Gold Bull., 2002, 35, 39-45.

6 P. Suchomel, L. Kvitek, R. Prucek, A. Panacek, A. Halder, S. Vajda and R. Zboril, Sci. Rep., 2018, 8, 4589.

7 C. Lin, K. Tao, D. Hua, Z. Ma and S. Zhou, Molecules, 2013, 18, 12609-12620.

8 A. S. K. Hashmi and G. J. Hutchings, Angew. Chem., Int. Ed., 2006, 45, 7896-7936.

9 K. J. M. Bishop, C. E. Wilmer, S. Soh and B. A. Grzybowski, Small, 2009, 5, 1600-1630.

10 C. Lourenco, M. Teixeira, S. Simões and R. Gaspar, Int. J. Pharm., 1996, 138, 1-12.

11 D. A. Walker, B. Kowalczyk, M. O. de la Cruz and B. A. Grzybowski, Nanoscale, 2011, 3, 1316-1344.

12 A. Alshammari and V. N. Kalevaru, Catalytic Application of Nano-Gold Catalyst, Supported Gold Nanoparticles as Promising Catalyts, InTech Open, 2016.

13 B. Gutmann, D. Cantillo and C. O. Kappe, Angew. Chem., Int. Ed., 2015, 54, 6688-6728.

14 N. G. Bastús, J. Comenge and V. Puntes, Langmuir, 2011, 27, 11098-11105.
15 T. Maruyama, Y. Fujimoto and T. Maekawa, J. Colloid Interface Sci., 2015, 447, 254-257.

16 S. Suarasan, M. Focsan, O. Soritau, D. Maniu and S. Astilean, Colloids Surf., B, 2015, 132, 122-131.

17 V. Kattumuri, M. Chandrasekhar, S. Guha, K. Raghuraman, K. V. Katti, K. Ghosh and R. J. Patel, Appl. Phys. Lett., 2006, 88, 153114.

18 X. Ma, Y. Xia, L. Ni, L. Song and Z. Wang, Spectrochim. Acta, Part A, 2014, 121, 657-661.

19 X. Wang, C. E. Egan, M. Zhou, K. Prince, D. R. G. Mitchell and R. A. Caruso, Chem. Commun., 2007, 3060-3062.

20 S. Kumar, K. S. Gandhi and R. Kumar, Ind. Eng. Chem. Res., 2007, 46, 3128-3136.

21 L. Shi, E. Buhler, F. Boué and F. Carn, J. Colloid Interface Sci., 2017, 492, 191-198.

22 M. Tran, R. DePenning, M. Turner and S. Padalkar, Mater. Res. Express, 2016, 3, 105027.

23 M. Kumari, A. Mishra, S. Pandey, S. P. Singh, V. Chaudhry, M. K. R. Mudiam, S. Shukla, P. Kakkar and C. S. Nautiyal, Sci. Rep., 2016, 6, 27575.

24 J. Rahbani, A. R. Behzad, N. M. Khashab and M. Al-Ghoul, Electrophoresis, 2012, 34, 405-408.

25 I. Lagzi and D. Ueyama, Chem. Phys. Lett., 2009, 468, 188192.

26 R. M. Walliser, F. Boudoire, E. Orosz, R. Tóth, A. Braun, E. C. Constable, Z. Rácz and I. Lagzi, Langmuir, 2015, 31, 1828-1834.

27 A. Gangula, R. Podila, M. Ramakrishna, L. Karanam, C. Janardhana and A. M. Rao, Langmuir, 2011, 27, 1526815274.

28 Z. D. Pozun, S. E. Rodenbusch, E. Keller, K. Tran, W. Tang, K. J. Stevenson and G. Henkelman, J. Phys. Chem. C, 2013, 117, 7598-7604.

29 H. Wu, X. Huang, M. Gao, X. Liao and B. Shi, Green Chem., 2011, 13, 651-658.

30 S. K. Das, C. Dickinson, F. Lafir, D. F. Brougham and E. Marsili, Green Chem., 2012, 14, 1322-1334. 